\title{
Overexpression of c-Met in bone marrow mesenchymal stem cells improves their effectiveness in homing and repair of acute liver failure
}

Kun Wang ${ }^{1,2+}$, Yuwen $\mathrm{Li}^{3+}$, Tiantian Zhu ${ }^{1,2}$, Yongting Zhang ${ }^{1}$, Wenting $\mathrm{Li}^{2}$, Wenyu Lin ${ }^{4}$, Jun $\mathrm{Li}^{1}$ and Chuanlong Zhu ${ }^{1 *}$ (D)

\begin{abstract}
Background: Transplantation of bone marrow-derived mesenchymal stem cells (BMSCs) has emerged as a novel therapy for acute liver failure (ALF). However, the homing efficiency of BMSCs to the injured liver sites appears to be poor. In this study, we aimed to determine if overexpression of c-Met in BMSCs could promote the homing ability of BMSCs to rat livers affected by ALF.

Methods: Overexpression of c-Met in BMSCs (c-Met-BMSCs) was attained by transfection of naive BMSCs with the lenti-c-Met-GFP. The impact of transplanted c-Met-BMSCs on both homing and repair of ALF was evaluated and compared with lenti-GFP empty vector transfected BMSCs (control BMSCs).

Results: After cells were transfected with the lenti-c-Met-GFP vector, the BMSCs displayed very high expression of c-Met protein as demonstrated by Western blot. In addition, in vitro transwell migration assays showed that the migration ability of c-Met-BMSCs was significantly increased in comparison with that of control BMSCs $(P<0.05)$, and was dependent on hepatocyte growth factor (HGF). Furthermore, rats with ALF that received transplanted cMet-BMSCs showed significantly improved homing ability to the injured liver; this was accompanied by elevated survival rates and liver function in the ALF rats. Parallel pathological examination further confirmed that transplantation of c-Met-BMSCs ameliorated liver injury with reduced hepatic activity index (HAl) scores, and that the effects of c-Met-BMSCs were more profound than those of control BMSCs.
\end{abstract}

Conclusions: Overexpression of c-Met promotes the homing of BMSCs to injured hepatic sites in a rat model of ALF, thereby improving the efficacy of BMSC therapy for ALF repair.

Keywords: c-Met, Bone marrow mesenchymal stem cells, Liver injury, Acute liver failure, Lentiviral vector

\section{Background}

Acute liver failure (ALF) is the rapid loss of liver function due to severe damage to the liver, with common causative factors including viruses (particularly hepatitis $\mathrm{B}$ and $\mathrm{C}$ ), toxins, prescribed drugs, and alcohol. ALF can lead to jaundice, coagulopathy, multiple organ failure, hepatic encephalopathy, and even death [1]. Currently,

\footnotetext{
* Correspondence: chuanlong@yahoo.com

${ }^{\dagger}$ Equal contributors

${ }^{1}$ Department of Infectious Disease, the First Affiliated Hospital with Nanjing

Medical University, Nanjing, China

Full list of author information is available at the end of the article
}

liver transplantation (LT) is considered the most effective therapy for this disease. However, its application for ALF is limited by a shortage of available donor organs and the procedure is invasive [2]. Bone marrow-derived mesenchymal stem cells (BMSCs) are multipotent stem cells that exhibit differentiation activity and significant potential for selfrenewal. Furthermore, these cells can differentiate into a variety of cell types, including osteoblasts, chondrocytes, adipocytes, and hepatocytes. It has also been reported that transfection of exogenous genes and consequent protein expression appear to be readily manipulated in BMSCs [3]. Considering the aforementioned advantages of BMSCs, 
they have been employed for the repair of damaged tissues or organs including the liver. In fact, BMSCs have been shown to be effective in the treatment of hepatic cirrhosis and liver failure. In addition, studies with BMSCs have demonstrated that they can repair ALF by regulating inflammatory responses and secreting trophic factors such as hepatocyte growth factor (HGF) and basic fibroblast growth factor (bFGF). However the ability of BMSCs to home to the injured liver has been reported as being poor. Consequently, this has posed challenges for development of their application [4]. Given the significant morbidity and mortality associated with ALF, there is an urgent need to enhance the homing capabilities of BMSCs in order to improve the efficacy of these potent cell types.

c-Met is encoded by the $M E T$ gene and belongs to the tyrosine protein kinase family. The c-Met protein is a member of the transmembrane tyrosine kinase receptor superfamily and has independent phosphorylation activity [5]. HGF is commonly known as the ligand of c-Met. The HGF/c-Met signaling pathway is considered to play an important role in the homing ability of BMSCs to the liver, which permits stem cell-mediated repair of the liver. BMSCs have been shown to influence both the differentiation of BMSCs into hepatocytes, and liver regeneration [6].

In this study, we aimed to establish c-Met-BMSCs by overexpression of $\mathrm{c}-\mathrm{Met}$, and to determine if c-MetBMSCs could promote homing of BMSCs to rat livers, thereby improving their capability for repairing ALF.

\section{Methods}

\section{Animals}

A total of seventy-two male Sprague-Dawley rats were purchased from the Animal Laboratory Center of Nanjing Medical University (Nanjing, China). Rats were aged 4 weeks, weighed $80-100 \mathrm{~g}$, and were used to isolate BMSCs so that an ALF rat model could be generated. All rats were maintained according to the experimental animal care and research protocol, which was approved by the First Affiliated Hospital of Nanjing Medical University (Nanjing, China). All experiments on rats were carried out in compliance with the guidelines of the Chinese Ethical Council.

\section{Isolation, culture, and validation of rat BMSCs}

Fresh rat BMSCs were isolated from male rats. Briefly, male rats were initially anesthetized by administration of $10 \%$ chloral hydrate at a dosage of $0.3 \mathrm{~mL} / 100 \mathrm{~g}$. The ends of the femur, tibia, and soft connective tissues were carefully removed to expose the intact bone marrow cavity, which was washed twice with normal saline solution, and collected into a centrifuge tube. Bone marrow material was centrifuged for $10 \mathrm{~min}$ at $180 \times \mathrm{g}$. The pellets were then dissolved in complete cell culture medium containing a low glucose solution of 89\% Dulbecco's modified Eagle's medium (DMEM; HyClone, Logan, USA), 10\% fetal bovine serum (FBS; Corning, New York, USA), and $1 \%$ penicillin/streptomycin (Biyuntian, China). The mixture was suspended in lymphocyte separation medium (Gibco, New York, USA) and centrifuged for $20 \mathrm{~min}$ at $710 \times \mathrm{g}$. The third layer was extracted, placed into a $10-\mathrm{cm}^{2}$ culture dish with complete culture medium, and cultured in an incubator at $37{ }^{\circ} \mathrm{C}$ at an atmosphere of $5 \% \mathrm{CO}_{2}$ with saturated humidity. BMSCs from the third passage were selected and then a total of $10^{5} / 100 \mu \mathrm{L}$ cells were labeled with different antibodies, including FITC-labeled anti-rat CD29, APC-labeled anti-rat CD45, PE-labeled anti-rat CD90, and anti-rat CD34 (Becton, Dickinson company, USA). The solution was subsequently stored on ice for $30 \mathrm{~min}$ in the dark. After washing with phosphate-buffered saline (PBS), the cells were subjected to analysis and validation of specific markers by flow cytometry.

\section{Animal model of acute liver failure}

Male rats were selected to generate the animal model of ALF as previously described [7]. In the protocol, rats received simultaneous intraperitoneal (i.p.) injections of Dgalactosamine (D-GalN) at $950 \mathrm{mg} / \mathrm{kg}$ body weight (Sigma, St. Louis, MO, USA) and lipopolysaccharide (LPS) at $10 \mu \mathrm{g} / \mathrm{kg}$ body weight (Sigma, St. Louis, MO, USA).

\section{Detection of HGF levels by enzyme-linked immunosorb- ent assay (ELISA)}

The heart, liver, spleen, lung, and kidneys of rats were collected at different time points $(0,24$, and $48 \mathrm{~h})$ postD-GalN/LPS injection; $50 \mathrm{mg}$ of each tissue was homogenized with $1.5 \mathrm{~mL}$ of lysis buffer (IS007-2, Cloud-clone Corp., China) and the lysate was collected to measure the HGF levels of all tissue samples using the HGF ELISA kit (Cloud-clone Corp., China), following the manufacturer's instructions.

\section{Construction and transfection of the lenti-c-Met-GFP and control lenti-GFP empty vectors}

For application of c-Met by polymerase chain reaction (PCR), a pair of primers were designed and synthesized as follows: Forward primer: 5'-GAGGATCCCCGGGTACCGGTCGCCACCATGAAGGCTCCCACCGCGCT GGCACCTGG-3'; Reverse primer: 5'-TCCTTGTAGTCCATACCTGTGTTCGCTTCGCCGTCAATGTTGTCT

TG-3'. The resulting c-Met cDNA was inserted into the lentiviral vector GV358 (sequence elements: Ubi-MCS3FLAG-SV40-EGFP-IRES-puromycin; Shanghai Genechem Company, China) to create the lentiviral vector, GV358-c-Met. We cotransfected the recombinant and two lentiviral helper plasmids (Helper1.0 and Helper2.0; Shanghai Genechem Company, China) into 293 T cells 
to generate the target lentivirus with an infectious viral titer of $2 \times 10^{8} \mathrm{TU} / \mathrm{mL}$, which was measured using a fluorescence assay method. In parallel, the control lentivirus was produced by cotransfecting the lenti-GFP empty vector GV358 with GFP but without c-Met, and two lentiviral helper plasmids (Helper1.0 and Helper2.0) into $293 \mathrm{~T}$ cells, and an infectious viral titer of $1 \times 10^{8}$ $\mathrm{TU} / \mathrm{mL}$ was obtained.

\section{Preparation of c-Met-BMSCs and control BMSCs}

To establish c-Met-BMSCs or control BMSCs, the lentic-Met-GFP or lenti-GFP empty vector was used to transfect naive BMSCs cells at passage $5(\mathrm{MOI}=100)$, respectively. Three days after infection, c-Met-BMSCs or control BMSCs were selected and cultured in cell culture medium containing $9 \mu \mathrm{g} / \mathrm{mL}$ puromycin for 2 weeks to generate stable cell lines (Patent no.201610662140.4). c-Met-BMSCs or control BMSCs were visualized under fluorescence microscopy to confirm a $100 \%$ fluorescence positive rate, and cells were screened and selected by puromycin.

\section{Western blot analysis of c-Met protein}

Western blot (WB) analysis was performed to examine levels of c-Met protein, in which the same number of cMet-BMSCs and control BMSCs (transfected with lentiGFP empty vector) were used to extract total protein samples. Proteins were then separated by sodium dodecyl sulfate-polyacrylamide gel electrophoresis (SDS-PAGE) and transferred onto nitrocellulose membranes as previously reported [6]. The c-Met protein was detected with the rabbit anti-c-Met (SP260) primary antibody (Santa Cruz Biotechnology, Santa Cruz, USA) with mouse antiactin antibody as control antibody (Santa Cruz Biotechnology, Santa Cruz, USA) for detection of the internal control protein, beta-actin. The secondary antibodies used in the WB analysis were a horseradish peroxidase (HRP)conjugated donkey anti-rabbit IgG and a HRP-conjugated ECL sheep anti-mouse IgG (GE Healthcare Biosciences, UK). The resulting blots were detected by enhanced chemiluminescence (ECL) using an Amersham ECL Western blot detection kit (GE Healthcare Biosciences, Pittsburgh, USA) and visualized under an imaging system.

\section{Cell migration assay}

The cell migration assay was performed in a transwell chamber (Corning, New York, USA) which contains a polyethylene terephthalate (PET) track-etched membrane with an $8.0-\mu \mathrm{m}$ pore; $0.5 \mathrm{~mL}$ suspensions of cMet-BMSCs or control BMSCs at a concentration of $1 \times$ $10^{5}$ cells $/ \mathrm{mL}$ were added to the top of the chamber layer. In the bottom chamber, different concentrations of murine HGF (Peprotech, USA) were used as a chemoattractant. The above cell transwell chambers were incubated in a humidified tissue culture incubator overnight at $37^{\circ}$ $\mathrm{C}$ and an atmosphere of $5 \% \mathrm{CO}_{2}$. After $24 \mathrm{~h}$, the cells were fixed and stained with $4 \%$ paraformaldehyde and $0.1 \%$ crystal violet, respectively. The migrated cells were observed, imaged, and counted within three fields under an optical microscope.

\section{Transplantation of c-Met-BMSCs and control BMSCs}

A total of 36 rats were randomly divided into three experimental groups: c-Met-BMSC group, control BMSC group, and normal saline (NS) group, with twelve rats in each group. The rat model of ALF was induced and generated as previously described by co-injection of $\mathrm{D}$ GalN/LPS. After $24 \mathrm{~h}$, rats in the c-Met-BMSC and control BMSC groups were given a transfusion of $1.0 \times 10^{7} /$ $\mathrm{kg}$ cells, suspended in $1 \mathrm{~mL}$ normal saline by vena caudalis injection, respectively. In the NS group, animals were injected with $1 \mathrm{~mL}$ of normal saline. The survival of rats in the three groups were observed and recorded daily. The blood samples of all rats were collected at different time points $(0,24,48$, and $72 \mathrm{~h})$ post-injection of D-GalN/LPS. The liver tissues of all the rats in the three groups were collected at 24,48 , or $72 \mathrm{~h}$ post-injection of D-GalN/LPS.

\section{Evaluation of liver necroinflammatory activity by serum ALT, AST, TBil, and hematoxylin-eosin staining}

The amount of inflammation in the liver was quantified by assessing serum levels of alanine transaminase (ALT), aspartate aminotransferase (AST), total bilirubin (TBil), and hematoxylin-eosin (HE) staining. Serum levels of ALT, AST, and TBil were detected using a microplate test kit from Nanjing Jiancheng bioengineering institute (Nanjing, China). Rat liver tissues obtained from all three experimental groups were fixed in $4 \%$ paraformaldehyde, embedded in paraffin, and sliced to a thickness of $4 \mu \mathrm{m}$. All slices were subsequently stained by HE. The pathological images were captured and grades of liver inflammation were assessed by hepatic activity index (HAI) grading following guidelines as previously described [8].

\section{Analysis of BMSC homing ability to rat liver}

To compare the homing efficiency of c-Met-BMSCs and control BMSCs to the injured rat liver, the dye DiR (AAT Bioquest, USA) was used to label both types of BMSCs, with or without c-Met overexpression; $1 \times 10^{6}$ cells from each group were transplanted into the rat with ALF through the vena caudalis. After $24 \mathrm{~h}$, migration of cells into the liver was examined using an in vivo imaging system (Animal Core Facility of Nanjing Medical University, Nanjing, China), and the homing efficiency was assessed by visualization of fluorescent intensity. 


\section{Statistical analysis}

The experiments in this study were performed with triplicate samples in each group. Statistical analysis was conducted using IBM Statistics SPSS, version 16.0 (SPSS, Chicago, IL, USA). Data are expressed as means \pm standard deviation (SD). The log rank test was used to compare survival rates among the three groups, and statistical analysis was carried out using one-way analysis of variance. $P$ values less than 0.05 were considered to be statistically significant.

\section{Results}

\section{Preparation and characterization of BMSCs and c-Met- BMSCs}

Seven to ten days after isolated BMSCs were cultured the cell morphology was examined under an inverted microscope. Monolayers of BMSCs were formed with cells displaying a spindle shape and arranged in radial concentric circles or with broom-like growth (data not shown). Next, we characterized and validated the BMSCs at passage 3 by flow cytometry using specific cell surface markers for BMSCs. As shown in Fig. 1a, 0.35\% of cells tested CD34-positive with $0.38 \%$ of cells being CD45-positive; $99.33 \%$ of the cells were CD29-positive and $99.81 \%$ were CD90-positive, indicating that the specific surface markers of the BMSCs had increased considerably in the prepared BMSCs. All results were consistent with the previous study by Li et al. [9]. Both the morphology shift and the fact that the surface markers of BMSCs were highly prevalent validated and confirmed the successful isolation of the BMSCs.

After naive BMSCs at passage 5 were transfected with the lenti-c-Met-GFP or lenti-GFP empty vector, and puromycin selection was completed, approximately $99 \%$ of these BMSCs were tested positive for green fluorescence as analyzed by fluorescence microscopy (Fig. 1b), indicating successful genetic modification of the BMSCs. We also observed that the expression of c-Met declined with an increase in the passage number of naive BMSCs (data not shown). Western blot analysis revealed that cMet protein was markedly overexpressed in c-MetBMSCs compared with control BMSCs (Fig. 1c), demonstrating the successful establishment of the c-Met-BMSC cell line with stable c-Met overexpression.

\section{Induction of HGF by the injured liver post-D-GaIN/LPS injection in rats}

As HGF is known to play an essential role in $\mathrm{HGF} / \mathrm{c}$ Met signaling-mediated repair of the injured liver, we examined the concentrations of HGF in the liver at 0, 24, and $48 \mathrm{~h}$ post-D-GalN/LPS injection. We also performed a parallel examination of HGF levels in other organs, including the heart, spleen, lung, and kidneys of the rat. The HGF concentrations at $0 \mathrm{~h}$ post-D-GalN/LPS injection were $4.89 \pm 0.08 \mathrm{ng} / \mathrm{mL}, 4.16 \pm 0.22 \mathrm{ng} / \mathrm{mL}, 1.29 \pm$ $0.02 \mathrm{ng} / \mathrm{mL}, 1.17 \pm 0.17 \mathrm{ng} / \mathrm{mL}$, and $4.24 \pm 0.145 \mathrm{ng} / \mathrm{mL}$ in the liver, heart, spleen, lung, and kidneys, respectively. We found that levels of HGF in the liver significantly increased after $24 \mathrm{~h}$, with a peak level at $48 \mathrm{~h}$ post-DGalN/LPS injection. However, there were no significant alterations in levels of HGF in the heart, spleen, lung, and kidneys of rats after D-GalN/LPS injection, which corroborates previous findings that HGF is induced by liver injury. As shown in Fig. 2, there was no statistical difference in HGF levels between these organs prior to D-GalN/LPS injection, whereas HGF levels in the liver
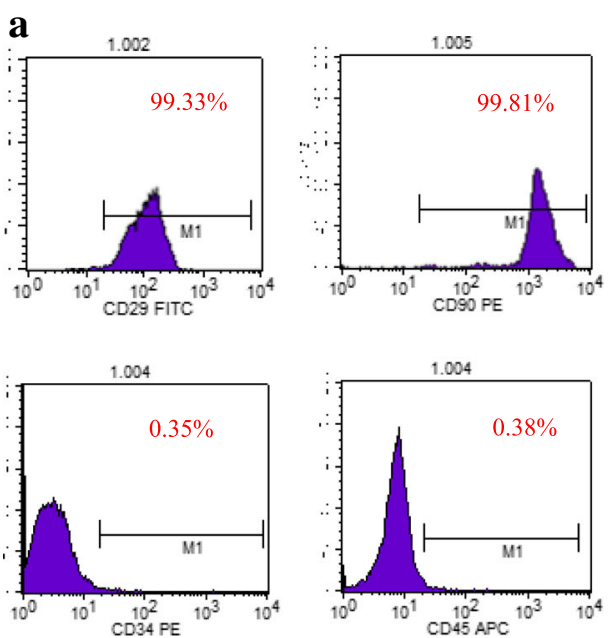

b

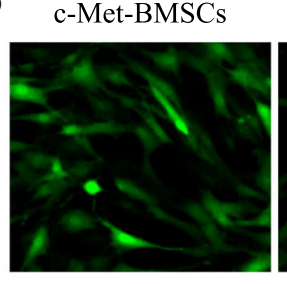

control BMSCs

C 12

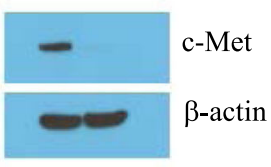

Lane 1: c-Met-BMSCs

Lane 2: control BMSCs

Fig. 1 Overexpression of c-Met in BMSCs transfected with lenti-c-Met-GFP vector. The BMSCs were stably transfected with lenti-c-Met-GFP or lenti-GFP empty vector, and then analyzed for c-Met protein expression. a Determination of the naive bone marrow-derived mesenchymal stem cell (BMSC) phenotypes by flow cytometry. b Transfection efficiency was detected by fluorescence microscopy ( $\times 400)$ in c-Met-BMSCs and control BMSCs. c Western blot analysis of c-Met protein expression in c-Met-BMSCs and control BMSCs 


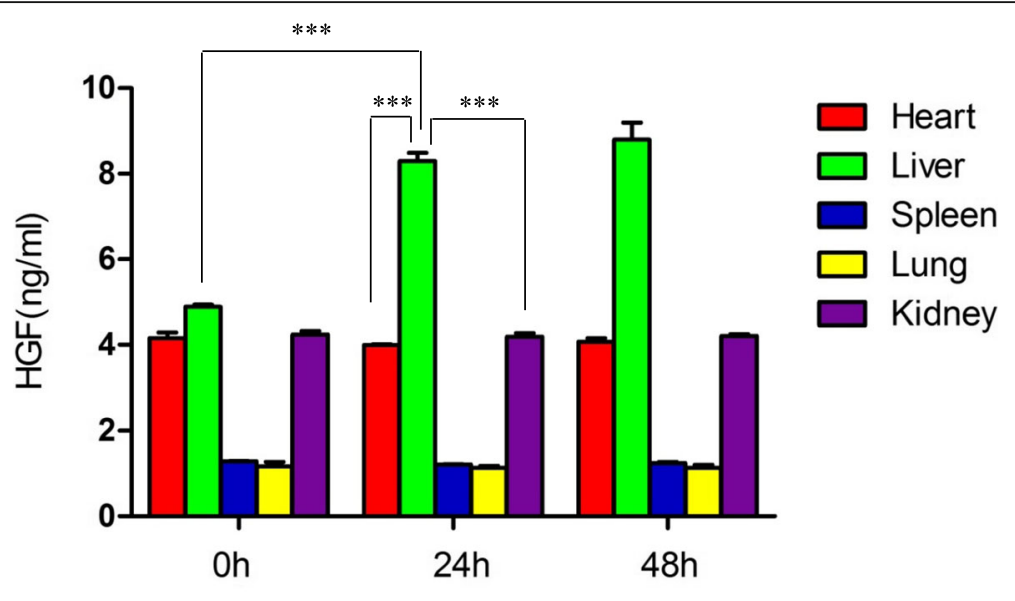

Hours after D-GalN/LPS injection

Fig. 2 The concentrations of HGF in the heart, liver, spleen, lung, and kidneys at different time points post-coinjection of D-GalN/LPS. The heart, liver, spleen, lung, and kidney tissues were collected at 0, 24, and $48 \mathrm{~h}$ post-coinjection of D-GalN/LPS; $50 \mathrm{mg}$ of each tissue was homogenized in $1.5 \mathrm{~mL}$ lysis buffer, and the lysate was collected for measurement of the concentrations of HGF by ELISA. Data are presented as mean \pm SD. ${ }^{* * * P}$ $<0.001$. D-GalN D-galactosamine, HGF hepatocyte growth factor, LPS lipopolysaccharide

were significantly higher compared with other organs at $24 \mathrm{~h}$ post-D-GalN/LPS injection $(P<0.001)$. These data indicate that there was induction of HGF through liver injury.

\section{Effects of c-Met overexpression on BMSC migration in- duced by HGF}

Next, we performed in vitro transwell migration assays to determine if the migration ability of c-Met-BMSCs could be improved in comparison with control BMSCs. The migrated BMSCs were visualized and counted under the microscope. The number of migrated cells in the cMet-BMSC group were $43.0 \pm 4.6,100.7 \pm 2.1,127.3 \pm$ $2.5,80.3 \pm 4.5,66.0 \pm 4.0$, and $61.0 \pm 3.6$, respectively, while the number of migrated cells in the control BMSC group were $43.0 \pm 2.0,43.3 \pm 5.0,44.33 \pm 1.2,43.3 \pm 2.1$, $44.0 \pm 3.0$, and $43.3 \pm 2.5$, respectively. As shown in Fig. $3 \mathrm{a}$, there was no significant difference in migrating cell numbers in the control BMSC group at different concentrations of HGF in the transwell chamber. However, at levels of HGF lower than $100 \mathrm{ng} / \mathrm{mL}$ the number of migrated cells in the c-Met-BMSC group increased in parallel with an increase in the concentrations of HGF (Fig. 3b). Interestingly, when the concentration of HGF was greater than $100 \mathrm{ng} / \mathrm{mL}$ the number of migrated cells actually decreased (Fig. 3b).

Enhanced ability of homing in c-Met-BMSCs to the liver of rats with ALF

As described above, enhanced in vitro migration of cMet-BMSCs may improve their homing ability to the injured liver. We therefore assessed effects of overexpressed c-Met protein on the homing capability of c-
Met-BMSCs to the liver of rats with ALF. Control BMSCs and c-Met-BMSCs were labeled with the dye $\mathrm{DiR}$ and then transplanted into the liver of rats with ALF. Fluorescence was released from DiR-labeled cells in affected organs, and the fluorescent intensity was measured using an imaging system (Fig. 4a). As shown in Fig. 4b, the fluorescent intensity was $(1.61 \pm 0.25) \times$ $10^{9}$ in the liver of ALF rats transplanted with control BMSCs, and $(3.22 \pm 0.80) \times 10^{10}$ in the liver of ALF rats transplanted with c-Met-BMSCs, which was significantly higher than that in the control BMSC group (this represented a fold-change of $19.84 \pm 1.71, P<0.001)$. These data demonstrated that c-Met-BMSCs could migrate more efficiently into the liver of ALF rats compared with control BMSCs.

\section{Effects of c-Met-BMSC transplantation on survival rates} and liver function in ALF rats

To evaluate the therapeutic effectiveness of c-MetBMSCs for treatment of ALF in rats, we established a rat model of ALF. The rats with ALF were randomly divided into three groups: the c-Met-BMSC group, the control BMSC group, and the NS group. The survival rates were $83.3 \%, 50 \%$, and $0 \%$ in the c-Met-BMSC, control BMSC, and NS groups, respectively (Fig. 5a). The ALF rats transplanted with c-Met-BMSCs had significantly higher survival rates than ALF rats treated with control BMSCs or NS $(P<0.05)$. In addition, we tested the serum levels of ALT, AST, and TBil at 0, 24, 48, and $72 \mathrm{~h}$ post-D-GalN/LPS injection. As shown in Fig. 5b-d, liver function started deteriorating $24 \mathrm{~h}$ after $\mathrm{D}-\mathrm{GalN} /$ LPS injection. However, there was no significant difference in serum levels of ALT, AST, and TBil among the 


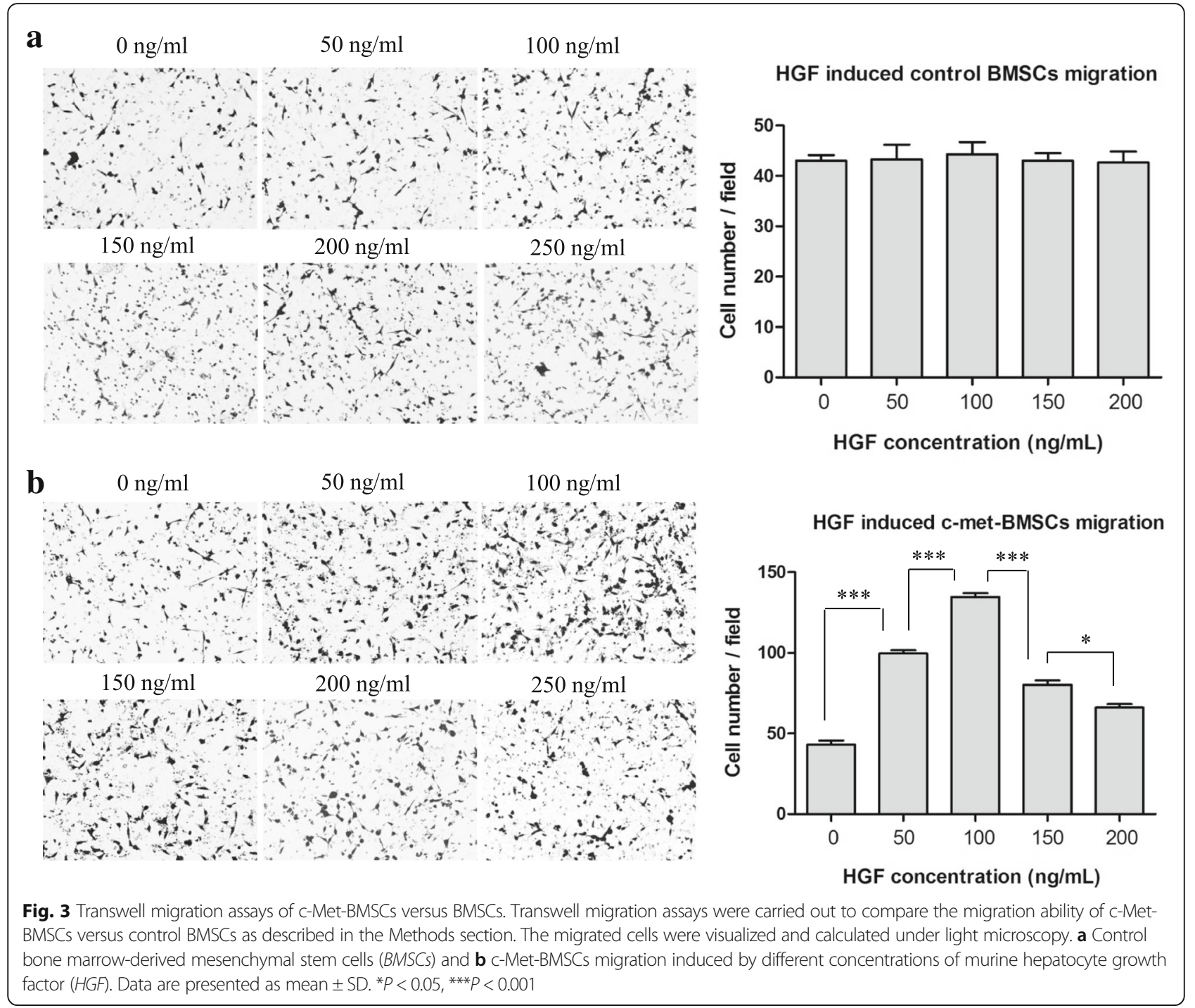

$\mathbf{a}$

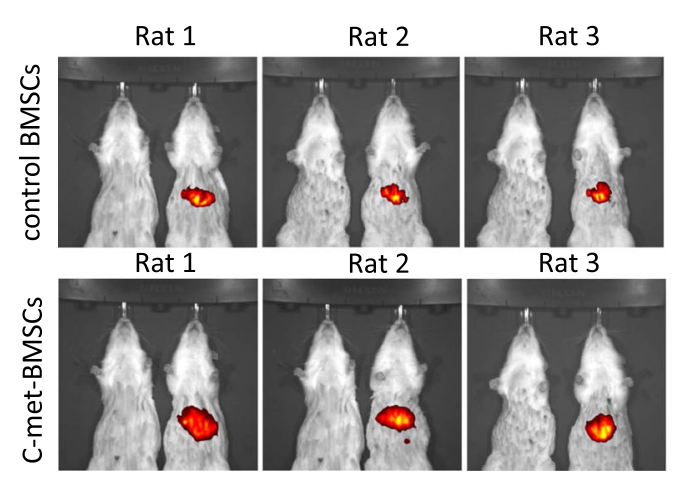

b

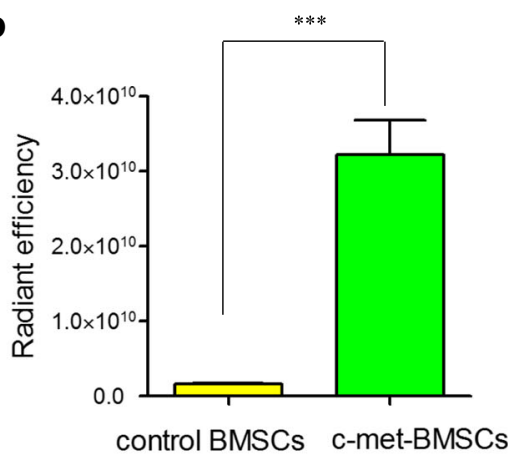

Fig. 4 Analysis of cell migration in ALF rats transplanted with c-Met-BMSCs using an in vivo imaging system. DiR dye was used to label the c-MetBMSCs and control BMSCs. The same amount of C-Met-BMSCs and control BMSCs were transplanted into ALF rats through the vena caudalis. After $24 \mathrm{~h}$ cells that had migrated to the injured liver were detected by an imaging system, and the fluorescent intensity was measured. a The fluorescent intensity of the liver tissues in rats transplanted with control bone marrow-derived mesenchymal stem cells (BMSCS) or c-Met-BMSCs. b The difference between the two groups was statistically significant. Data are presented as mean \pm SD. ${ }^{* * *} P<0.001$ 


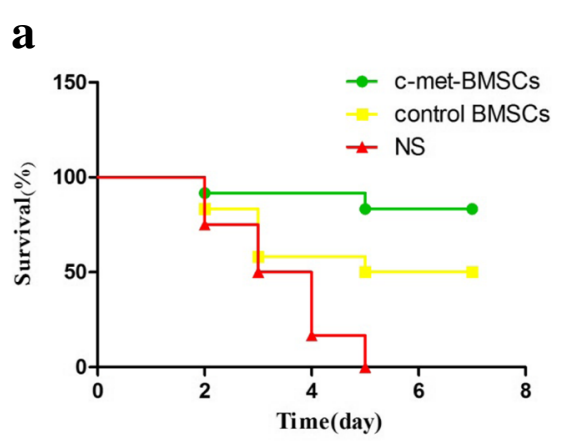

c

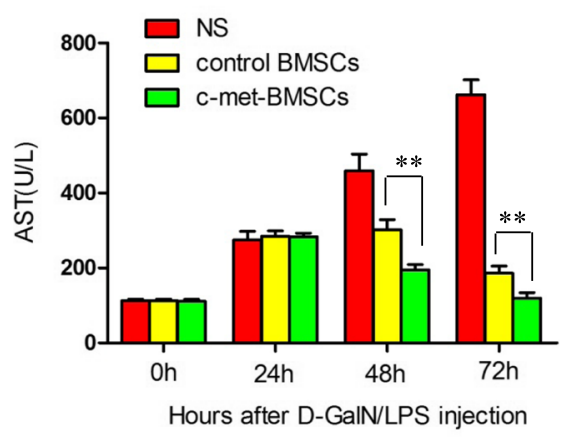

b

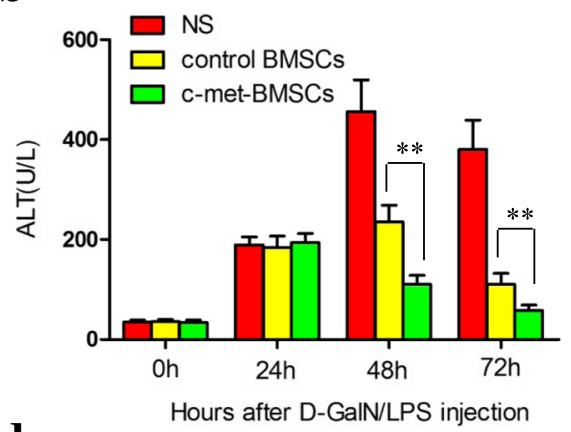

d

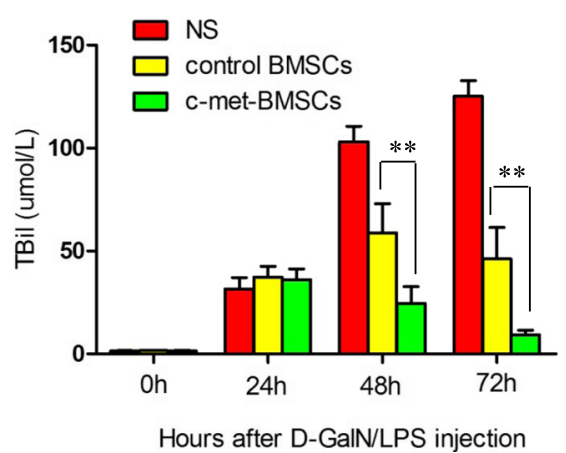

Fig. 5 Effects of c-Met-BMSC transplantation on the survival rates and liver function in ALF rats. A total of 54 rats were randomly divided into three groups: c-Met-BMSC, control BMSC, and NS groups ( $n=12$ in each group). The rats were injected with D-GalN/LPS to induce ALF. After 24 h, the c-Met-BMSC and control BMSC groups were given a transfusion of $1.0 \times 10^{7} / \mathrm{kg}$ cells suspended in $1 \mathrm{~mL}$ of normal saline (NS) respectively, while the NS group was given $1 \mathrm{~mL}$ of NS. The blood samples were collected at 0,24, 48 and $72 \mathrm{~h}$ post-D-GalN/LPS injection. a The survival rates in the c-MetBMSC, control BMSC, and NS groups; levels of serum $\mathbf{b}$ alanine transferase (ALT), $\mathbf{c}$ aspartate aminotransferase (AST), and $\mathbf{d}$ total bilirubin (TBi) in treatment of ALF rats. Data were expressed as mean \pm SD. ${ }^{*} P<0.05,{ }^{* *} P<0.01$, and ${ }^{* * *} P<0.001$. BMSC bone marrow-derived mesenchymal stem cell, D-GalN D-galactosamine, LPS lipopolysaccharide

three groups. The serum levels of ALT, AST, and TBil were significantly lower in the c-Met-BMSCs group compared with NS group, at $48 \mathrm{~h}$ or $72 \mathrm{~h}$ after D-GalN/ LPS injection $(P<0.001)$. Moreover, significant differences in serum levels of ALT, AST, and TBil were observed between c-Met-BMSCs and control BMSCs groups at both 48 and $72 \mathrm{~h}$ post-D-GalN/LPS injection $(P<0.01)$.

\section{Effects of c-Met-BMSC transplantation on liver function} through evaluation of liver pathology in rats with ALF We further examined whether c-Met-BMSC transplantation could improve repair of ALF as assessed by liver histology. Liver histology was conducted by HE staining at the indicated times. More than 10 low-power field microscopic examinations were carried out for each slide. We did not observe hepatic necrosis in any of the three groups at $24 \mathrm{~h}$ post-D-GalN/LPS injection. We observed moderate hepatic necrosis in the NS and control BMSC groups, whereas only mild hepatic necrosis was observed in the c-Met-BMSC group at $48 \mathrm{~h}$ post-DGalN/LPS injection. At $72 \mathrm{~h}$ after D-GalN/LPS injection, moderate or severe hepatic necrosis was observed in the
NS and control BMSC groups, whereas only sporadic (spotty) hepatic necrosis was observed in the c-MetBMSC group (Fig. 6b). Furthermore, the pathological scores were determined using the HAI by blinding of expert pathologists. As shown in Fig. 6c, there were significant differences in HAI scores among the three groups at both 48 and 72 h post-D-GalN/LPS injection $(P<0.001)$. Interestingly, HAI scores in the c-Met-BMSC group were significantly lower than those in the control BMSC group at 48 and $72 \mathrm{~h}$ post-D-GalN/LPS injection $(P<0.01)$. These findings strongly suggest that c-Met-BMSCs improved the liver function in ALF induced by D-GalN/LPS and, in some of the cases, even resulted in reversal of the disease allowing rats to recover from ALF.

\section{Discussion}

Transplantation of mesenchymal stem cells (MSCs), liver progenitor cells (LPCs), and hematopoietic stem cells (HSCs) has emerged as a promising therapy to treat various forms of liver disease mainly due to the capacity of these cell types to promote liver regeneration [10, 11]. However, only a small proportion of stem cells appear to be able to migrate to the liver, which largely results in 


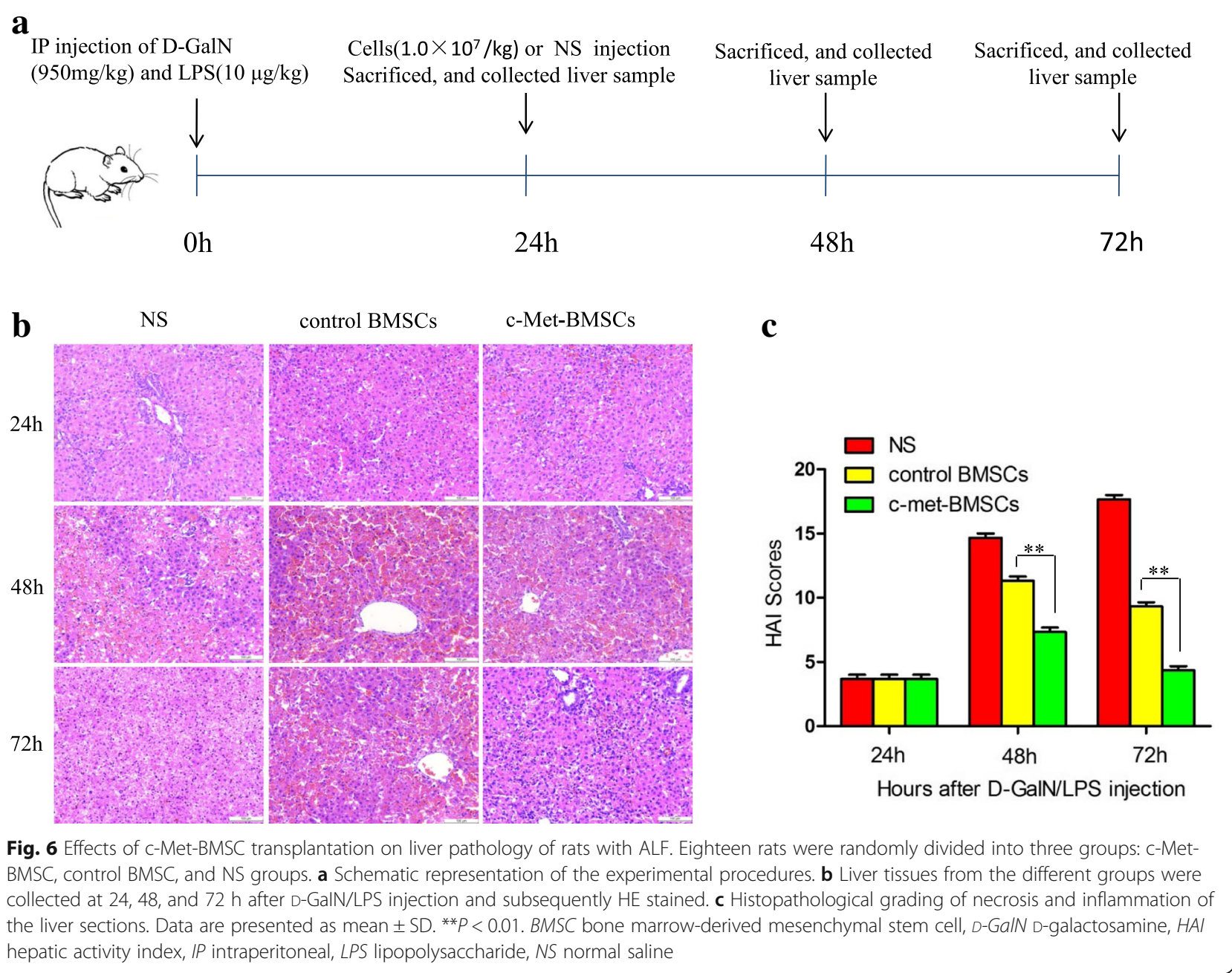

decreased efficacy of their therapeutic use. The main novel findings in this study can be summarized as follows: 1) overexpression of c-Met significantly increased the migration ability of BMSCs in vitro; 2) c-Met-BMSCs homed into ALF-affected rat livers more efficiently than control BMSCs; 3) transplantation of c-Met-BMSCs resulted in significantly increased survival rates and liver function of rats with ALF compared with administration of control BMSCs; and 4) transplantation of c-Met-BMSCs greatly ameliorated liver injury, and the effects of c-Met-BMSCs exceeded those of control BMSCs.

In this study, the ALF rat model was generated by intraperitoneal injection of D-GalN/LPS as previously reported [7]. In recent years, many studies have shown that transplantation of BMSCs could repair injured tissues including those in the liver $[12,13]$. Indeed, BMSC transplantation has been considered as a promising approach for the treatment of ALF $[9,14]$, and has been attributed to the ability of the cells to differentiate into primary hepatocytes and promote liver regeneration $[15,16]$. Furthermore, Salomone and colleagues have reported that transplantation of adipose tissue-derived mesenchymal stem cells (ASCs) was effective in treating acetaminophen-induced liver injury. ASCs appear to engraft in the injured liver where they enhance hepatocyte regeneration and inhibit stress and inflammatory signaling [17]. Furthermore, these cells can produce a range of cytokines and growth factors, including insulin-like growth factor-1, vascular endothelial growth factor-1, epidermal growth factor, HGF, and keratinocyte growth factor $[18,19]$, which have been shown to promote hepatocyte regeneration in situ, suppress inflammatory responses, and inhibit apoptosis of hepatocytes. However, the repair efficacy of BMSC transplantation has been found to be poor [20] mainly due to the low homing efficiency of BMSCs into the injured sites of the liver [4].

HGF is a potent hepatic mitogen produced by liver mesenchymal cells. Zhu et al. [6] have reported that the level of HGF in the liver is significantly upregulated in the rat model of ALF. Our finding that HGF significantly increased after liver injury induced by D-GalN/LPS injection in rats is consistent with previous results. Since HGF has been found to strongly inhibit apoptosis of 
hepatocytes, elevated levels of HGF may improve the survival rate of mice with ALF. Further studies have shown that HGF also plays an essential role in stimulating liver regeneration against $\operatorname{ALF}[21,22]$. c-Met is known as the receptor for HGF, and the HGF/c-Met signaling pathway has been demonstrated to participate in different cellular processes such as apoptosis and cell proliferation. Indeed, stimulation of this pathway has shown profound antiapoptosis, antioxidation, and cell proliferation-promoting effects [23, 24]. Moreover, enhancement of HGF/c-Met signaling can promote regeneration of hepatocytes in response to $\operatorname{ALF}[25,26]$. Recently, a number of studies have shown that the HGF/ c-Met signaling pathway can promote cell migration [27, 28]. Therefore, we hypothesized that overexpression of c-Met could promote the homing capabilities of BMSCs to the rat livers affected by ALF, and consequently improve the therapeutic efficacy of BMSC transplantation for the treatment of ALF.

Extensive studies have demonstrated that gene therapy is a promising approach for the treatment of many forms of diseases, and may improve patient care, survival, and outcomes [29, 30]. Viral vectors such as lentivirus, adenovirus, and adeno-associated viral vectors are widely used, mainly because they are highly efficient in introducing target genes into host cells. Compared with adenovirus and adeno-associated viral vectors, lentivirus possesses the ability to transport larger gene fragments thus ensuring earlier protein expression [31]. As a result, lentivirus is an attractive gene delivery system and is widely used in gene therapy [32]. The lentivirus expression vector, originated from the human immunodeficiency virus (HIV), is capable of infecting almost all mammalian cell types. Considering the advantages of the lentivirus as a high-efficiency gene delivery system for gene therapy [33], the lentivirus expression vector was selected in this study to deliver the c-Met gene into BMSCs. Our results validated the stable expression of cMet protein in BMSCs after transfection.

Notably, the levels of HGF in liver tissue were significantly upregulated at $24 \mathrm{~h}$ and $48 \mathrm{~h}$ after induced liver injury in the ALF rat model. When the c-Met-BMSC and control BMSC transplantation groups were compared, we found that ALF rats in the c-Met-BMSC transplantation group experienced better therapeutic effects as assessed by survival rates, markers for liver function, and liver pathology. It is plausible that induction of the HGF/c-Met signaling pathway led to augmented cMet-BMSC migration to the liver and, as a result, a greater number of c-Met-BMSCs were able to home into the rat liver. This theory is supported by our study results, which showed increased migration of BMSCs by in vitro transwell assays. Furthermore, we were able to demonstrate homing capabilities of c-Met-BMSCs to the injured liver compared with control BMSCs using an in vivo imaging system.

Our study does have some limitations, as we could not exclude the possibility that cytokines such as SDF-1 might also be involved in the migration of c-MetBMSCs [34-36], and an increase in the paracrine effects of c-Met-BMSCs may be responsible for a better effect in comparison with control BMSCS. Further studies are underway in our laboratory to characterize c-MetBMSC-secreted cytokines in cell culture. In addition, stable expression of a proto-oncogene such as c-Met may lead to increased risk of tumorigenesis. Therefore, it is necessary to regulate exogenous expression of cMet in the c-Met modified BMSCs by using the tetracycline-on (Tet-On) system [37] prior to its application in future clinical studies.

\section{Conclusion}

In conclusion, our results indicated that overexpression of c-Met promotes homing of BMSCs to the injured liver of rats with ALF, thereby improving the efficacy of BMSC therapy for the repair of ALF.

\begin{abstract}
Abbreviations
ALF: Acute liver failure; ALT: Alanine transaminase; ASC: Adipose tissuederived mesenchymal stem cell; AST: Aspartate aminotransferase; bFGF: Basic fibroblast growth factor; BMSC: Bone marrow-derived mesenchymal stem cell; D-GalN: D-Galactosamine; DIR: Deep red fluorescence; DMEM: Dulbecco's modified Eagle's medium; ECL: Enhanced chemiluminescence;

ELISA: Enzyme-linked immunosorbent assay; FBS: Fetal bovine serum;

HAl: Hepatic activity index; HE: Hematoxylin-eosin; HGF: Hepatocyte growth factor; HIV: Human immunodeficiency virus; HRP: Horseradish peroxidase; HSC: Hematopoietic stem cell; LPC: Liver progenitor cell;

LPS: Lipopolysaccharide; LT: Liver transplantation; MSC: Mesenchymal stem cell; NS: Normal saline; PET: Polyethylene terephthalate; SD: Standard deviation; SDS-PAGE: Sodium dodecyl sulfate-polyacrylamide gel electrophoresis; TBil: Total bilirubin; Tet-On: Tetracycline-on; WB: Western blot
\end{abstract}

\section{Acknowledgements}

We thank Mrs. Yaping Han (the First Affiliated Hospital of Nanjing Medical University, Nanjing, China) for excellent technical assistance.

\section{Funding}

This work was supported by the National Natural Science Foundation of China (No. 81271713), the Gilead Sciences Research Scholars Program in Liver Disease-Asia and the Key Medical Talents Fund of Jiangsu Province (ZDRCA2016007)

\section{Availability of data and materials}

All data generated or analyzed supporting conclusions are included in the current manuscript.

\section{Authors' contributions}

$\mathrm{CZ}$ co-conceived the study. KW and YL performed the majority of the experiments related to the differentiation protocol and endpoint assessments. TZ, $Y Z$, and WL contributed to the experimental execution of BMSC cell culture, Western blot, and flow cytometry. CZ and JL performed data analysis. KW and $\mathrm{CZ}$ co-wrote the manuscript. All authors contributed to the study design, and revised and approved the manuscript.

\section{Ethics approval}

All animal experiments were approved by the Animal Care and Use Committee of the Nanjing Medical University (protocol number: NJMU13-021). 


\section{Consent for publication}

Not applicable.

\section{Competing interests}

The authors declare that they have no competing interests.

\section{Publisher's Note}

Springer Nature remains neutral with regard to jurisdictional claims in published maps and institutional affiliations.

\section{Author details}

'Department of Infectious Disease, the First Affiliated Hospital with Nanjing Medical University, Nanjing, China. ${ }^{2}$ Department of Infectious Disease, Anhui Provincial Hospital, Anhui Medical University, Hefei, China. ${ }^{3}$ Department of Pediatrics, the First Affiliated Hospital with Nanjing Medical University, Nanjing, China. ${ }^{4}$ Liver Center and Gastrointestinal Division, Department of Medicine, Massachusetts General Hospital, Harvard Medical School, Boston, Massachusetts, USA

Received: 16 March 2017 Revised: 13 June 2017 Accepted: 19 June 2017 Published online: 05 July 2017

\section{References}

1. Cai Y, Zou Z, Liu L, Chen S, Chen Y, Lin Z, Shi K, Xu L, Chen Y. Bone marrow-derived mesenchymal stem cells inhibits hepatocyte apoptosis after acute liver injury. Int J Clin Exp Pathol. 2015;8(1):107-16.

2. Basto ST, Villela-Nogueira CA, Tura BR, Coelho HS, Ribeiro J, Fernandes ES, Schmal AF, Victor L, Luiz RR, Perez RM. Risk factors for long-term mortality in a large cohort of patients wait-listed for liver transplantation in Brazil. Liver Transpl. 2011;17(9):1013-20.

3. Kazuhiko K, Kimianori N, Takashi T. BDNF gene-modified mesenchymal stem cells promote functional recovery and reduced infarct size in the rat middle cerebral artery occlusion model. Mol Ther. 2004;9(2):189-97.

4. Gao J, Dennis JE, Muzic RF, Lundberq M, Caplan A. The dynamic in vivo distribution of bone marrow-derived mesenchymal stem cells after infusion. Cells Tissues Organs. 2001;169(1):12-20.

5. Gherardi E, Birchmeier W, Birchmeier C, Vande WG. Targeting MET in cancer: rationale and progress. Nat Rev Cancer. 2012;12(2):89-103.

6. Zhu C, Li Y, Li W, Wu Q, Gao R. Gene transfer of c-met confers protection against D-galactosamine/lipopolysaccharide-induced acute liver failure. Dig Dis Sci. 2012:57(4):925-34.

7. Zheng S, Yang J, Tang Y, Yang J, Shao Q, Guo L, Liu Q. Effect of bone marrow mesenchymal stem cells transplantation on the serum and liver HMGB1 expression in rats with acute liver failure. Int J Clin Exp Pathol. 2015; 8(12):15985-92.

8. Theise ND. Liver biopsy assessment in chronic viral hepatitis: a personal, practical approach. Mod Pathol. 2007;20(1):S3-14

9. Li D, Fan J, He X, Zhang X, Zhang Z, Zeng Z, Ruan M, Cai L. Therapeutic effect comparison of hepatocyte-like cells and bone marrow mesenchymal stem cells in acute liver failure of rats. Int J Clin Exp Pathol. 2015;8(1):11-24

10. Tsolalo E, Yannaki E. Stem cell-based regenerative opportunities for the liver: state of the art and beyond. World J Gastroenterol. 2015;21(43):12334-50.

11. Lorenzini S, Gitto S, Grandini E, Andreone P, Bernardi M. Stem cells for end stage liver disease: how far have we got? World J Gastroenterol. 2008;14(29): 4593-99.

12. Uccelli A, Moretta L, Pistoia V. Mesenchymal stem cells in health and disease. Nat Rev Immunol. 2008;8(9):726-36.

13. Phinney DG, Prockop DJ. Concise review: mesenchymal stem/multipotent stromal cells: the state of transdifferentiation and modes of tissue repaircurrent views. Stem Cells. 2007;25(11):2896-902.

14. Yuan SF, Jiang T, Sun LH, Zheng RJ, Cao GQ, Ahat NZ, Zhang YX. Use of bone mesenchymal stem cells to treat rats with acute liver failure. Genet Mol Res. 2014;13(3):6962-80.

15. Houlihan DD, Newsome PN. Critical review of clinical trials of bone marrow stem cells in liver disease. Gastroenterology. 2008;135(2):438-50.

16. Thorqeirsson SS, Grisham JW. Hematopoietic cells as hepatocyte stem cells: a critical review of the evidence. Hepatology. 2006;43(1):2-8.

17. Salomone F, Barbagallo I, Puzzo L, Piazza C, Li VG. Efficacy of adipose tissuemesenchymal stem cell transplantation in rats with acetaminophen liver injury. Stem Cell Res. 2013;11(3):1037-44.
18. van Poll D, Parekkadan B, Cho CH, Berthiaume F, Nahmias Y, Tilles AW Yarmush ML. Mesenchymal stem cell-derived molecules directly modulate hepatocellular death and regeneration in vitro and in vivo. Hepatology. 2008:47(5):1634-43.

19. Chen L, Tredget EE, Wu PY, Wu Y. Paracrine factors of mesenchymal stem cells recruit macrophages and endothelial lineage cells and enhance wound healing. PLoS One. 2008;3(4):e1886.

20. Peng L, Xie DY, Lin BL, Liu J, Zhu HP, Xie C, Zheng YB, Gao ZL. Autologous bone marrow mesenchymal stem cell transplantation in liver failure patients caused by hepatitis B: short-term and long-term outcomes. Hepatology. 2011:54(3):820-8.

21. Böhm F, Köhler UA, Speicher T, Werner S. Regulation of liver regeneration by growth factors and cytokines. EMBO Mol Med. 2010;2(8):294-305.

22. Nejak-Bowen K, Orr A, Bowen Jr WC, Michalopoulos GK. Conditional genetic elimination of hepatocyte growth factor in mice compromises liver regeneration after partial hepatectomy. PLoS One. 2013;8(3):e59836.

23. Xiao GH, Jeffers M, Bellacosa A, Mitsuuchi Y, Vande Woude GF, Testa JR. Anti-apoptotic signaling by hepatocyte growth factor/Met via the phosphatidylinositol 3-kinase/Akt and mitogen-activated protein kinase pathways. Proc Natl Acad Sci U S A. 2001;98(1):247-52.

24. Valdés-Arzate A, Luna A, Bucio L, Licona C, Clemens DL, Souza V, Hernandez E, Kershenobich D, Gutiérrez-Ruiz MC, Gómez-Quiroz LE. Hepatocyte growth factor protects hepatocytes against oxidative injury induced by ethanol metabolism. Free Radic Biol Med. 2009:47(4):424-30.

25. Huh CG, Factor VM, Sánchez A, Uchida K, Conner EA, Thorqeirsson SS. Hepatocyte growth factor/c-met signaling pathway is required for efficient liver regeneration and repair. Proc Natl Acad Sci U S A. 2004;101(13):447782.

26. Schmidt C, Bladt F, Goedecke S, Brinkmann V, Zschiesche W, Sharpe M, Gherardi E, Birchmeier C. Scatter factor/hepatocyte growth factor is essential for liver development. Nature. 1995;373(6516):699-702.

27. Shibata S, Miwa T, Wu HH, Levitt P, Ohyama T. Hepatocyte growth factor-cmet signaling mediates the development of nonsensory structures of the mammalian cochlea and hearing. J Neurosci. 2016;36(31):8200-9.

28. Leung $E$, Xue A, Wang Y, Rougerie P, Sharma VP, Eddy R, Cox D, Condeelis J. Blood vessel endothelium-directed tumor cell streaming in breast tumors requires the HGF/C-Met signaling pathway. Oncogene. 2017;36(19):2680-92.

29. Zhu CL, Li YW, Gao RT. Gene therapy for acute liver failure. Curr Gene Ther. 2010:10(2):156-66.

30. Booth C, Gaspar HB, Thrasher AJ. Treating Immunodeficiency through HSC gene therapy. Trends Mol Med. 2016;22(4):317-27.

31. Kelly MJ, O'Keeffe GW, Sullivan AM. Viral vector delivery of neurotrophic factors for Parkinson's disease therapy. Expert Rev Mol Med. 2015;17:e8.

32. McCarron A, Donnelley M, McIntyre C, Parsons D. Challenges of up-scaling lentivirus production and processing. J Biotechnol. 2016;240:23-30.

33. Rubinson DA, Dillon CP, Kwiatkowski AV, Sievers C, Yang L, Kopinja J, Rooney DL, Zhang M, Ihriq MM, McManus MT, Gertler FB, Scott ML, Van Parijs L. A lentivirus based system to functionally silence genes in primary mammalian cells, stem cells and transgenic mice by RNA interference. Nat Genet. 2013;33(3):401-6.

34. Xiao Ling K, Peng L, Jian Feng Z, Wei C, Wei Yan Y, Nan S, Cheng Qi G, Zhi Wei W. Stromal derived factor-1/CXCR4 axis involved in bone marrow mesenchymal stem cells recruitment to injured liver. Stem Cells Int. 2016; 2016:8906945.

35. Liu N, Patzak A, Zhang J. CXCR4-overexpressing bone marrow-derived mesenchymal stem cells improve repair of acute kidney injury. Am J Physiol Renal Physiol. 2013:305(7):F1064-73.

36. Ma HC, Shi XL, Ren HZ, Yuan XW, Ding YT. Targeted migration of mesenchymal stem cells modified with CXCR4 to acute failing liver improves liver regeneration. World J Gastroenterol. 2014;20(40):14884-94.

37. Benabdellah K, Muñoz $P$, Cobo M, Gutierrez-Guerrero A, Sánchez-Hernández S, Garcia-Perez A, Anderson P, Carrillo-Gálvez AB, Toscano MG, Martin F. Lent-On-Plus lentiviral vectors for conditional expression in human stem cells. Sci Rep. 2016;6:37289. 